\title{
Templated Three-Dimensional Engineered Bone Matrix as a Model for Breast Cancer Osteolytic Bone Metastasis Process
}

\author{
Manman Sun' \\ Ke Huang' \\ Xueshi Luo $\mathbb{( D}^{2,3}$ \\ Hong $\mathrm{Li}^{1}$ \\ 'Department of Materials Science and \\ Engineering, College of Chemistry and \\ Materials Science, Jinan University, \\ Guangzhou, Guangdong, People's \\ Republic of China; ${ }^{2}$ The First Affiliated \\ Hospital of Jinan University, Jinan \\ University, Guangzhou, Guangdong, \\ People's Republic of China; ${ }^{3}$ Department \\ of Chemistry, College of Chemistry and \\ Materials Science, Jinan University, \\ Guangzhou, Guangdong, People's \\ Republic of China
}

Purpose: Bone metastasis is one of the common causes of death relative to breast cancer. However, the evolvement of bone niche in cancer progression remains poorly understood. A three-dimensional (3D) engineered bone matrix was developed as an effective biomimetic model to explore the mechanism relative to bone cancer metastasis.

Methods: In the study, a 3D engineered bone matrix was developed via cell biomineralization templated by a biomimetic collagen template. The process of bone metastasis relative to breast cancer was investigated by co-culturing breast cancer MDA-MB-231-GFP cells with pre-osteogenic MC3T3-E1 cells on the 3D bone matrix.

Results: A typical bone matrix was obtained, where mineralized collagen fibers were packed into the bundle to form a 3D engineered bone matrix. As the cancer cells were invading along the way vertical to the alignment of mineralized collagen fiber, the bone matrix gradually became thinner, accompanied with the erosion of Col I and the loss of calcium and phosphorus. As a result, the disassembled structure of mineralized collagen fiber was observed, which may be attributed to osteolytic bone metastasis.

Conclusion: An engineered 3D bone-like matrix was successfully prepared via cell mineralization, which can act as a model for bone metastasis process. The study revealed mineralized collagen fiber disassembled at nanoscale relative to breast cancer cells.

Keywords: biomimetic models, breast cancer, MDA-MB-231-GFP cells, bone metastasis

\section{Introduction}

Breast cancer is the most common cancer diagnosed in women and the second leading cause of cancer-related deaths. ${ }^{1}$ Usually, advanced breast cancer metastasizing to the bone in about $70 \%$ of patients disrupts physiological bone remodeling and ultimately leads to poor clinical prognosis. ${ }^{2-4}$ It is characterized by fast growth and progressive bone destruction through osteolysis, which means the loss of bone mineral density. ${ }^{5}$ Osteolytic bone metastasis is extremely aggressive to patients, leading to pathologic fractures, bone pain, and other complications that seriously threaten patients' life. ${ }^{6,7}$ However, the development of effective treatment strategies for cancer bone metastases has been hindered by the lack of appropriate models that can recapitulate the biology of human diseases. Much attention has been paid to the molecular and cellular events that drive breast cancer bone metastasis and a considerable understanding of how cancer cell influences osteoblast behavior during metastasis in vivo and in vitro. ${ }^{4,8,9}$ According to most studies, ${ }^{10-13}$ metastatic breast cancer cells colonize within the bone marrow space, interrupting the
Correspondence: Xueshi Luo; Hong Li Tel +8602085223062

Fax +860208522327I

Email 1254914942@q9.com;

tlihong@jnu.edu.cn 


\section{Graphical Abstract}

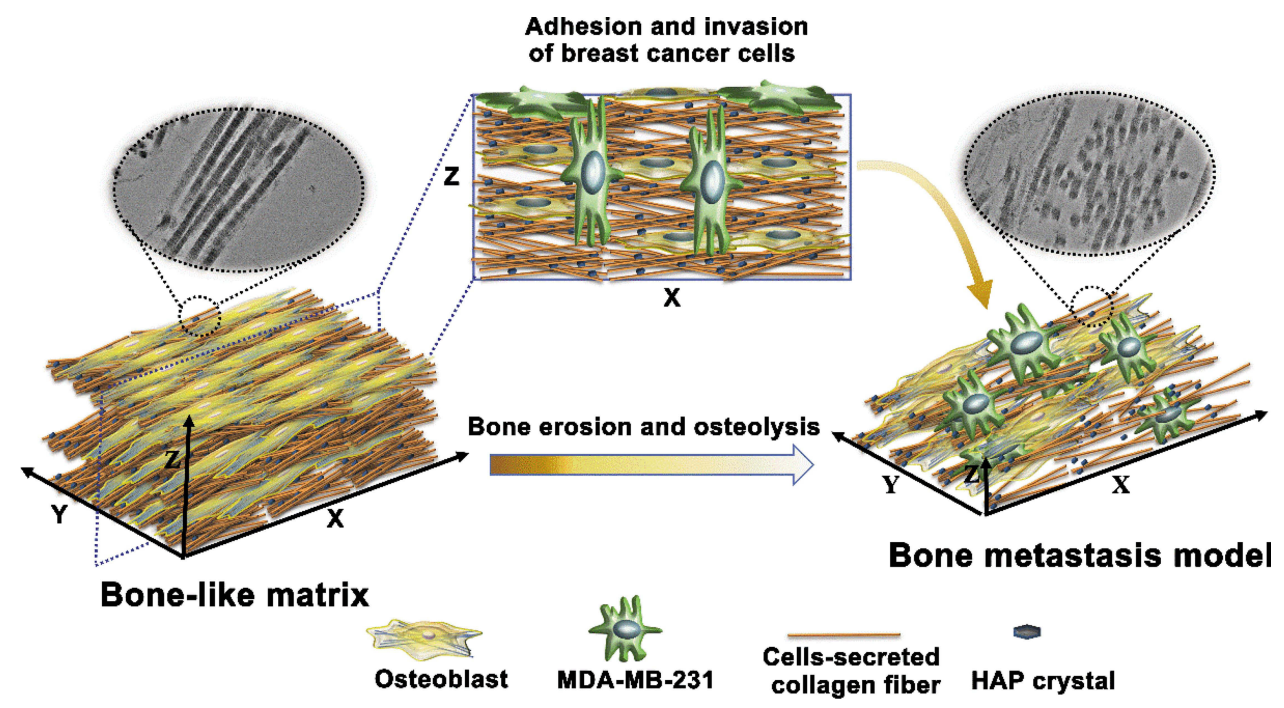

normal bone remodeling process by activating the differentiation of osteoclasts (bone-resorbing cells) and inhibiting the activity of osteoblasts (bone-forming cells). ${ }^{14-16}$ Indeed, different models have been developed to study this interaction and investigate treatment strategies to inhibit it. ${ }^{17-19}$ However, it is not clear what happens to the bone extracellular matrix (ECM) after cancer cells homing to the bone, which is a key step in the process of osteolytic bone metastasis. This deficiency seriously hinders the development of new therapeutic methods. Typically, it is mainly due to the complexity of the natural bone environment and the lack of reproducible and controllable experimental models to simulate pathophysiological events, especially the bone osteolysis process. ${ }^{16,20}$

The bone structure comprises mainly structural collagen fibrils, hydroxyapatite (HAP) nanocrystals and water. It is the organization of the densely packed collagen matrix that guides the arrangement of the mineral crystallites. ${ }^{21,22}$ From a view of materials, bone possess multiple-level hierarchical structure, while mineralized collagen fibril is its basic building block. Generally, loadbearing bone tissue commonly has mineralized collagen fibrils organized in parallel arrays, which are then arranged in an array pattern at the next level of hierarchy. ${ }^{23}$ Recapitulating bone-like structures using synthetic approaches has been explored due to the demanding requirements of bone regeneration engineering. Recently, increasing studies in vitro models, which mimicked the bone microenvironment, involving the metastasis of breast cancer to bone were performed. For instance, mineralized type I collagen (Col I) fibrils acted as a model that allowed studying tumor cell behavior, and the study showed intrafibrillar HAP decreased breast cancer cell adhesion forces. ${ }^{4}$ Polymer scaffolds with calcium phosphates could provide a biologically relevant environment, increased cell-cell and cell-matrix interactions in native bone. ${ }^{4,16} \beta$ tricalcium-phosphate ( $\beta$-TCP) scaffolds with a customized interconnecting channel structure could enable an appropriate three-dimensional (3D) mimetic bone matrix microenvironment for the growth of metastatic neuroblastoma cells. $^{23,24}$ The 3D in vitro culture model designed to replicate the tumor microenvironment may provide a new frontier for cancer research, and its cell growth, morphology and genetic characteristics are closer to cancer in vivo. ${ }^{25,26}$ However, most of the studies have tested the effect of isolated or combined natural bone components on breast cancer cells that do not allow identification of the effect of natural bone tissue with the specific structure on tumor cells. Let alone, mimicking the resident cells' behavior drives tumor-associated osteolysis. To fully understand the interaction between breast tumor cells and the bone microenvironment where osteoblast, various biomolecules participate in forming a vicious circle and promoting tumor cell and osteolytic bone metastases, a 3D in vitro tissue-like model both compositionally, structurally and functionally similar to the bone matrix is required. 
In the process of osteogenesis, highly self-assembled oriented collagen fibrils provide a natural template for inorganic mineral deposition, thus realizing the hierarchical and ordered structure of inorganic minerals in the bone matrix. ${ }^{27,28}$ The formation of bone tissue is a multilevel ordered structure process of mineralization of organic template with the participation of osteoblasts. Histologically, the template is an osteoid structure assembled by cells secreting collagen with an ordered liquid crystal structure. ${ }^{18}$ Once osteoid mineralization occurs, it means bone formation. We hypothesize that the mineralized matrix produced by osteoblasts, which formed the organic and inorganic components of bone, is a suitable in vitro model system for bone metastasis research that mimics in vivo conditions. What is more, the resident osteoblast can also provide a vital role in the tumor-associated osteolysis processing.

However, for conventional cell-based fabrication, no scaffold provides enough ability to produce osteoid matrix, which templates to form appropriate 3D meso-structures of bone tissue. With the development of tissue engineering, as we know, oriented collagen fibrous scaffolds could induce cells to secrete ECM with oriented structure. ${ }^{29-32}$ This statement provides an applicable way to fabricate biomimetic structures. Herein, an osteoid-like template was constructed via shear stress, and an engineered cell-mineralized model mimicking natural bone was constructed on the template, where typical mineralized collagen fibrils were packed in a bone-like way. Utilizing this 3D model, breast cancer cell invasion was studied, and the role of the mineralized matrix in the process was assessed. This study provides valuable evidence and a contribution to understanding the theoretical mechanism of breast cancer bone metastasis and provides an engineered 3D tumor model for exploring new therapy.

\section{Materials and Methods Preparation and Characterization of Osteoid Collagen Template}

The osteoid collagen template was manufactured using shear stress according to the previous study. ${ }^{30}$ Briefly, Col I (extracted from Sprague Dawley rat tails) was dissolved in acetic acid solution $(0.5 \mathrm{~mol} / \mathrm{L})$ to prepare $120 \mathrm{mg} / \mathrm{mL}$ collagen solution. The collagen solution was carefully poured on a glass plate and then another glass plate was pressed and pulled using directional and uniform sliding shear. The collagen templates were neutralized in the ammonia environment for $30 \mathrm{~min}$, washed with ultrapure water 3 times and dried at room temperature. Then, these templates were cut into disks (diameter $=14 \mathrm{~mm}$, thickness $=150-200 \mu \mathrm{m})$ and put into 24-well plates. The samples were sterilized with $\mathrm{Co}^{60}$ irradiation and used for subsequent relevant experiments.

Scanning electron microscope (SEM, PXL30, Philips, Netherlands) was used to observe the surface micromorphology of the collagen template. All samples were treated by spraying platinum and examined at a voltage of $15 \mathrm{kV}$. X-ray diffractometer (XRD, Miniflex 600, Rigaku, Japan) and Fourier transform infrared spectrometer (FTIR spectrometer, EQUINOX55, Bruker, Germany) were used to analyze the composition of the collagen template. XRD test conditions were as follows: $\mathrm{Cu}-\mathrm{K} \alpha$ target, voltage of $36 \mathrm{kV}$, tube flow of $20 \mathrm{~mA}$, scanning range from $5^{\circ}$ to $60^{\circ}$, scanning speed of $4^{\circ} \%$ min. The FTIR test conditions were as follows: the scanning wave number range was $4000 \mathrm{~cm}^{-1} \sim 500 \mathrm{~cm}^{-1}$.

\section{Cell Culture}

Mouse pre-osteoblast cells MC3T3-E1 (MC3T3-E1 Subclone 14, CC-Y2056, Shanghai Biological Technology Co., Ltd., China) were seeded on the oriented collagen templates at a density of $1 \times 10^{4}$ cells and cultured with alpha minimum essential medium ( $\alpha$-MEM, Gibco, USA), supplemented with $10 \%(\mathrm{v} / \mathrm{v})$ fetal bovine serum (FBS, Gibco, USA) and 1\% (v/v) penicillin/streptomycin solution (Biological Industries, Israel). The cell culture was maintained in an incubator (Heraeus, Germany) at $37^{\circ} \mathrm{C}$ and $5 \% \mathrm{CO}_{2}$.

\section{D Bone Matrix Formation}

Once the cells reached confluence, the medium was replaced by the mineralized medium ( $\alpha$-MEM supplemented with $50 \mathrm{mg} / \mathrm{mL}$ ascorbic acid (Aladdin, China) and $5 \mathrm{mM} \beta$-glycerophosphate (Sigma, USA)). After culturing for 4 weeks, cell-produced ECM formed on the collagen template. Then the 3D matrix including Col I and minerals was fetched from the osteoid collagen template, which was named as engineered bone matrix.

\section{In vitro Bone Metastases}

The bone matrix obtained by cell mineralization was used to simulate in vitro bone tissue microenvironment. Green fluorescent protein (GFP)-transfected human breast cancer cells (MDA-MB-231-GFP, Procell CL-0642, Procell Life Science \& Technology Co., Ltd., China) and MC3T3-E1 cells were directly co-cultured in vitro. Firstly, briefly, 
$1 \times 10^{4}$ MC3T3-E1 cells were added to the prepared engineered bone matrix and cultured for 3 days to form a new layer of active cells on the surface. Then, $5 \times 10^{3}$ MDA-MB -231-GFP cells were seeded for co-cultivation. The ratio of the number of MDA-MB-231-GFP cells to the number of MC3T3-E1 cells is 1:2. The mixed medium $(45 \%$ DMEM $+45 \% \quad \alpha$-MEM $+10 \%$ FBS $+1 \%$ penicillin/streptomycin solution) of dulbecco's modified eagle medium (DMEM, Gibco, USA) and $\alpha$-MEM was used for cocultivation.

\section{Characterization of Engineered Bone Matrix: SEM, FTIR Analysis and XRD Analysis}

The engineered bone matrices were washed 3 times with phosphate buffer saline (PBS), fixed with $2.5 \%$ glutaraldehyde for 4 hours, then washed with PBS for 3 times and dehydrated with gradient alcohol $(50 \%, 60 \%, 70 \%, 80 \%$, $90 \%, 100 \%$ ) for 10 minutes each gradient. After drying, the samples were sprayed with platinum and observed by SEM.

The matrices were removed from the collagen templates, washed 3 times with ultrapure water, and dried at room temperature. FTIR and XRD analysis were employed to analyze the composition of the matrices. The operation procedure is described above.

\section{Mineral Deposit in the Engineered Bone Matrix: Inductively Coupled Plasma Mass Spectrometry and Von Kossa (VK) \\ Staining}

The content variation of calcium $(\mathrm{Ca})$ and phosphorus $(\mathrm{P})$ elements were detected using inductively coupled plasma mass spectrometry (ICP-MS). The matrices were dried and crushed. $10 \mathrm{~mL}$ nitric acid and $0.1 \mathrm{~g}$ of each matrix of the two samples were put in ampoules, respectively. Then they were heated to $100^{\circ} \mathrm{C}$ in the fume hood, and $0.3 \mathrm{~mL}$ hydrofluoric acid (HF) was added after a lot of yellow smoke dissipated. They were heated until the solution was clarified and then were diluted to $100 \mathrm{~mL}$ with ultrapure water. The concentrations of $\mathrm{Ca}$ and $\mathrm{P}$ elements in the matrices were analyzed by an induced coupled plasmaatomic emission spectrophotometer (Perkin-Elmer, Optima 2000DV).

To confirm the mineral formation in the matrices by VK (LEAGENE, China) staining, the samples were fixed at $4{ }^{\circ} \mathrm{C}$ with $4 \%$ paraformaldehyde for 2 hours and then washed with PBS. When the fixation was completed, VK silver solution was added and irradiated under the ultraviolet lamp for 15-30 minutes. After being cleaned by ultrapure water, samples were treated with sodium hyposulfite for $2 \mathrm{~min}$. Then, photos were taken under the microscope for analysis.

\section{Col I Immunofluorescence Staining}

Laser scanning confocal microscopy (LSCM) was used to observe the cells' morphologies and 3D distribution. After in vitro mineralization was completed, the samples were fixed with $4 \%$ paraformaldehyde for 15 minutes, washed 3 times with PBS, and penetrated with $0.1 \%$ Triton- 100 for 5 minutes. Then, $1 \%$ bull serum albumin (BSA, Sigma, USA) solution was applied for an hour to seal off the nonspecific binding site. All antibodies were diluted with PBS with $1 \%$ BSA. In order to observe the formation of Col I secreted by cells at different times, the Col I was immunostained. Since MC3T3-E1 was derived from mice, to distinguish it from the collagen template (extracted from rat tail), $300 \mu \mathrm{L}$ Col I monoclonal antibody (1:250 diluted, ab21286, Abcam, Britain) of mice was used to incubate with the samples overnight and avoid light at $4^{\circ} \mathrm{C}$. Then the samples were stained with $500 \mu \mathrm{L}$ Alexa Fluor 630 labeled anti-mouse secondary antibody (1:500 diluted, IgG H\&L $\left(\mathrm{Cy} 3{ }^{\circledR}\right)$ ex652 nm/em668 nm, Abcam, Britain) originated from goat for 1 hour after being washed with PBS for 3 times. Finally, the samples were incubated with 4',6-diamidino-2-phenylindole (DAPI ex358 nm/em $461 \mathrm{~nm}$, Invitrogen, USA) for 5 minutes to complete the nuclear staining and photographed for observation.

LSCM was used to observe cell morphologies and variation of collagen content after what was being co-cultured. Col I immunofluorescence staining was performed on the bone matrix models co-cultured with MDA-MB-231-GFP for 1,4 and 7 days, respectively. After the staining, the variation of collagen during bone metastasis of breast cancer cells and the invasion morphologies of MDA-MB-231GFP cells were analyzed by z-axis laminar scanning.

The average orientation angle of cell-produced Col I matrix fibers and breast cancer cells were calculated by taking the mean of the orientation angle of each fiber or breast cancer cell. The angular distribution of the cellproduced Col I matrix fibers and breast cancer cells was determined based on the relative frequency of cellproduced Col I matrix fibers and breast cancer cells 
(classified into bins of $20^{\circ}$ angles) of three LSCM images with analysis software (WCIF Image J).

Field emission transmission electron microscopy (FETEM, JEM-2100F, JEOL, Japan) was used to analyze the microscopic morphologies of the ultrafine structure of the matrices, including mineralized collagen fibrils and HAP in the osteoblast matrices before and after breast cancer cell metastasis, and the inorganic crystal phase was analyzed by selective electron diffraction. The samples were dispersed into acetone solution after ultrapure water cleaning. Then the solution was fully diluted and deposited on the copper-screen substrate. Then the morphologies of the samples were photographed and observed. At the same time, the inorganic phase was photographed and analyzed by Fourier transform selected area electron diffraction (SAED). For further ultrastructural characterization by TEM, the mineralized matrices for 1, 4 and 7-day coculturing were fixed with $2 \%$ glutaraldehyde and dehydrated through a series of graded ethanol dilutions. Samples were embedded in epoxy resin. Ultrathin sections (80-nm-thick) were cut with an ultramicrotome, placed on formvar-coated nickel grids and stained with uranyl acetate. The specimens were investigated using the FETEM.
For the observation of the templated osteoblast matrices before and after bone metastasis, the samples were cleaned with ultra-pure water, embedded in resin and sliced, then placed on copper-screen substrate and photographed for observation by TEM. FTIR and ICPMS also were used according to the method shown above.

\section{Statistical Analysis}

All statistical analyses were performed using analysis of variance (ANOVA) and Tukey's post hoc test, in which differences were considered significant for $\mathrm{ns}=\mathrm{p}>0.05 /$ $* \mathrm{p}<0.05 / * * \mathrm{p}<0.01 / * * * \mathrm{p}<0.001$.

\section{Results}

\section{Feasibility of 3D Engineered Bone Matrix}

As seen in Figure 1A, the templated 3D bone matrix was constructed via cell mineralization. SEM was used to analyze the morphology of the prepared collagen template. The template shows an oriented fibrous structure (Figures 1B and 1b-1), similar to that of osteoid tissue. After 4 weeks in vitro cell culture, a layer of fibrous matrix can be observed on the template, where the nanofibrils orderly assembled along the same alignment (Figure 1C),
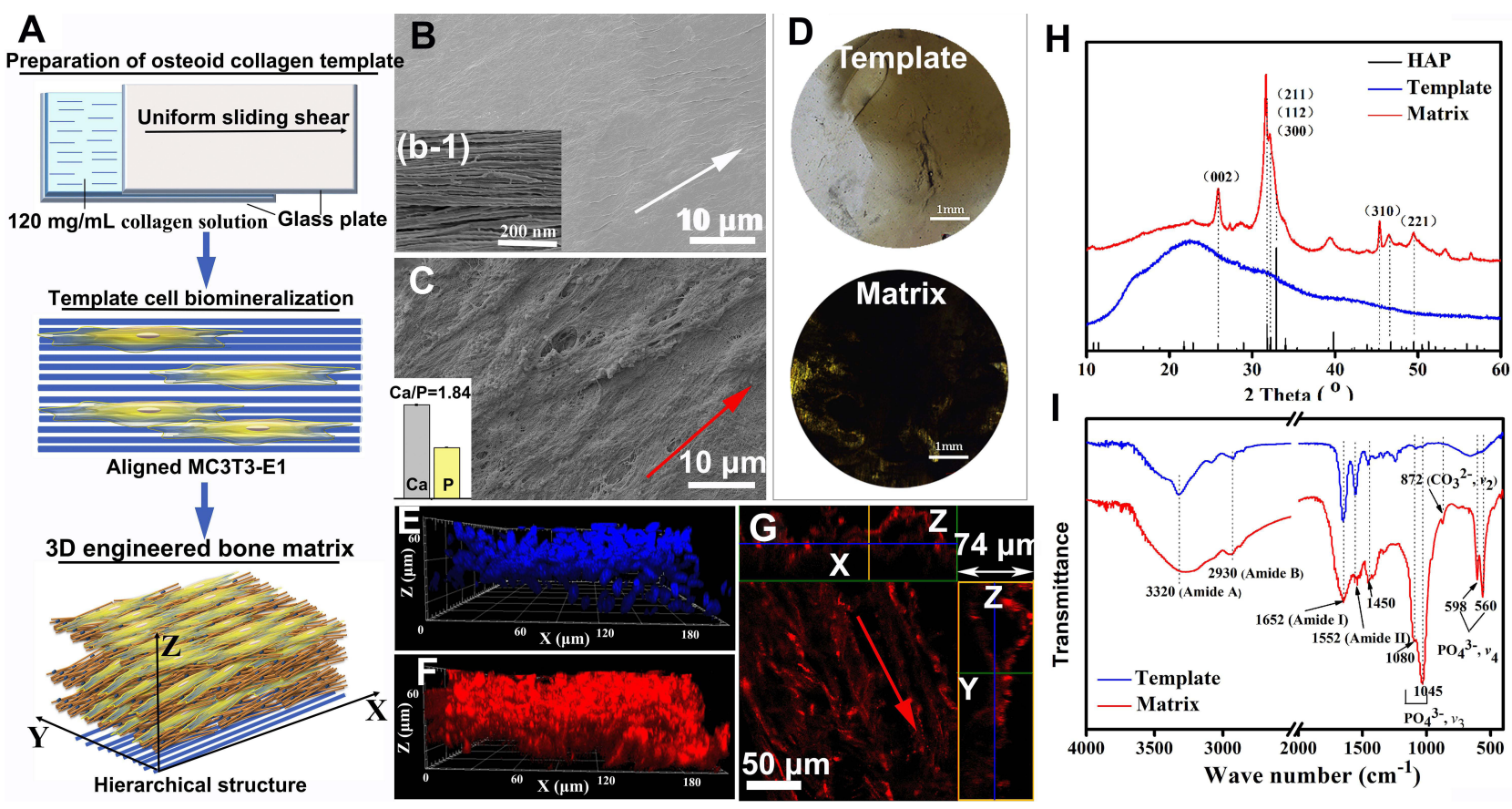

Figure I Preparation and characterization of osteoid collagen template and the 3D engineered bone matrix. (A) Schematic diagram of the preparation of osteoid collagen template, templated cell biomineralization and the 3D engineered bone matrix formation; (B) SEM images of the osteoid collagen template and the illustration (b-I) on the lower left is an enlarged view of collagen fibers in the collagen template. The white arrows indicate the orientation of the osteoid collagen template (C) SEM images the 3D engineered bone matrix produced via osteoblast mineralization and the illustration (c-I) on the lower left is the contents of Ca and P determined by ICP-MS; (D) VK staining of the collagen template and the 3D engineered bone matrix; $(\mathbf{H})$ XRD patterns and FTIR spectra (I) of osteoid collagen template and bone-like matrix. (E) 3D distribution of osteoblasts in the matrix (cell nucleus in blue). (F) 3D distribution of cell-secreted Col I of bone-like matrix (in red). (G) 2D oriented morphologies of Col I in the bonelike matrix. The red arrows indicate the orientation of the bone-like matrix. 
as the oriented collagen template. An inserted content of $\mathrm{Ca}$ and $\mathrm{P}$ determined by ICP-MS shows the occurrence of HAP, altogether with FTIR and XRD in Figure $1 \mathrm{H}$ and I, respectively. Obvious HAP characteristic diffraction peaks in the formed matrix are observed, which are mainly manifested as $26^{\circ}(002), 32^{\circ}$ (211) (112) (300) and $39^{\circ}$ (310) crystal plane diffraction peaks. ${ }^{26,27}$ In addition, the characteristic diffraction peaks of HAP in the matrix show larger half-peak widths, which may be caused by the small size and low-crystallinity of HAP crystals formed via cell mineralization.

FTIR spectra of the samples in Figure 1I has peaks at the $3320 \mathrm{~cm}^{-1}$ (stretching vibration $v_{\mathrm{N}-\mathrm{H}}$ peak of amide A band), $2930 \mathrm{~cm}^{-1}$ (stretching vibration $v_{\mathrm{N}-\mathrm{H}}$ and $v_{\mathrm{C}-\mathrm{H}}$ peaks of amide B band), $1652 \mathrm{~cm}^{-1}$ ( $v_{\mathrm{N}-\mathrm{H}}$ peak of amide I), $1552 \mathrm{~cm}^{-1}$ (weaker in-plane $\delta_{\mathrm{N}-\mathrm{H}}$ peak of amide II), and $1454-1240 \mathrm{~cm}^{-1}$ (the characteristic sequence peaks of Proline-Glycine-Hydroxyproline), which indicates the existence of a large amount of organic collagen. Compared with the collagen template, the characteristic peak of the phosphate group $\left(\mathrm{PO}_{4}{ }^{3-}\right)$ appears at about $1045 \mathrm{~cm}^{-1}$, which includes the triple asymmetric stretching peak of $\mathrm{PO}_{4}{ }^{3-}$ at $1080 \mathrm{~cm}^{-1}, 1065 \mathrm{~cm}^{-1}$, and $1020 \mathrm{~cm}^{-1}$. The sharp absorption peak at $1015 \mathrm{~cm}^{-1}$ is due to the overlap of the absorption peaks of $-\mathrm{NH}_{2}$ and $\mathrm{PO}_{4}{ }^{3-}$, and the absorption peak of the $\mathrm{Ca}-\mathrm{O}$ bond at about $506 \mathrm{~cm}^{-1}$ also proves the existence of calcium phosphate in the matrix. ${ }^{33,34}$

VK staining was used to analyze the inorganic calcium salt deposition in the matrix qualitatively. Figure 1D shows the results of VK staining in the collagen template and the formed matrix. It can be seen that a large number of black deposits appear in the matrix, which means that the inorganic $\mathrm{Ca}$ salts are distributed in the matrix.

Combined with the analysis results of the $\mathrm{Ca}$ and $\mathrm{P}$ content by ICP-MS, the ratio of $\mathrm{Ca} / \mathrm{P}$ in the matrix is 1.84 (as shown in the inserted image in Figure 1C), which is slightly higher than the ratio of 1.67 in the normal human bone, indicating that the group of $\mathrm{PO}_{4}{ }^{3-}$ would be substituted by other groups. Energy Dispersive Spectrometer (EDS) mapping analysis of $\mathrm{Ca}$ and $\mathrm{P}$ elements in matrix microregions (as shown in Figure S1 in Supporting Information) also proves the existence of inorganic minerals and their uniform distribution among the formed matrix.

According to the above results, the ECM secreted by cells contains orientated collagen fibrils and an inorganic product of HAP. The complex structure of like-bone tissue cannot be separated from the participation of cells.
Figure $1 \mathrm{E}$ is the nuclear staining image of MC3T3-E1 cells, which shows that osteoblasts grew in multiple layers with a thickness of about $60 \mu \mathrm{m}$. LSCM analysis was performed on Col I with specific immunofluorescence staining (Figure $1 \mathrm{~F}$ and $\mathrm{G}$ ), where $\mathrm{Z}$-axis stratification was used for continuous photography and cell-produced Col I matrix was reconstructed in 3D. The matrix contains a large amount of cell-produced Col I matrix (Figure 1F), and the collagen fibrils are continuously densely banded with thickness of $74 \mu \mathrm{m}$ in z-axis. In addition, a picture of the $\mathrm{z}$-axis slice was selected to observe and analyze the internal structure of the matrix (Figure 1G), with the horizontal green box corresponding to the $\mathrm{x}-\mathrm{Z}$ axis section and the longitudinal yellow box corresponding to the $y-z$ axis section in Figure 1G. The cell-produced collagen fibrils are assembled in an aligned orientation. It means that the template conducted the oriented assembly of cellproduced collagen during cell biomineralization.

According to the above results, the osteoblast-produced matrix is compositionally and morphologically similar to the in vivo bone tissue microenvironment. We can note the matrix as an engineered bone matrix, which may meet the basic requirements for the in vitro study of breast cancer bone metastasis.

\section{Analysis of Bone Metastasis}

To simulate the process of bone metastasis, the 3D engineered bone matrix was taken as a metastasis model to observe the invasion of cancer cells after 1, 4 and 7 days of culture as shown in Figure 2. On the first day, the cancer cells were mainly distributed at the top of the matrix model and the morphology of single cell was clearly visible (Figure 2A and $\mathrm{G}$ ), while the few cells grew into the matrix. Then, the cancer cells are in conjunction with each other to cover the surface of the bone matrix, while a few cells have penetrated through the matrix to the bottom after 4-day culture (Figure 2B and H). MDA-MB -231 cells (in green) proliferated rapidly, invaded throughout the collagen matrix on the 7th day (Figure 2C and I). On the 7th day, MDA-MB-231 cells (in green) proliferated massively, and the collagen matrix became less and less. The cancer cells and the matrix fused and could not be distinguished (Figure 3I and L).

According to the immunostaining of $\mathrm{Col} \mathrm{I}$ as shown in Figure 2 in red, the thickness of the Col I matrix gradually decreased while the MDA-MB-231 cells proliferated. Figure $2 \mathrm{~J}-\mathrm{L}$ corresponds to the $\mathrm{x}-\mathrm{y}, \mathrm{x}-\mathrm{z}$, and $\mathrm{y}-\mathrm{z}$ axes sections in the 3D reconstructed model. We could find that the 


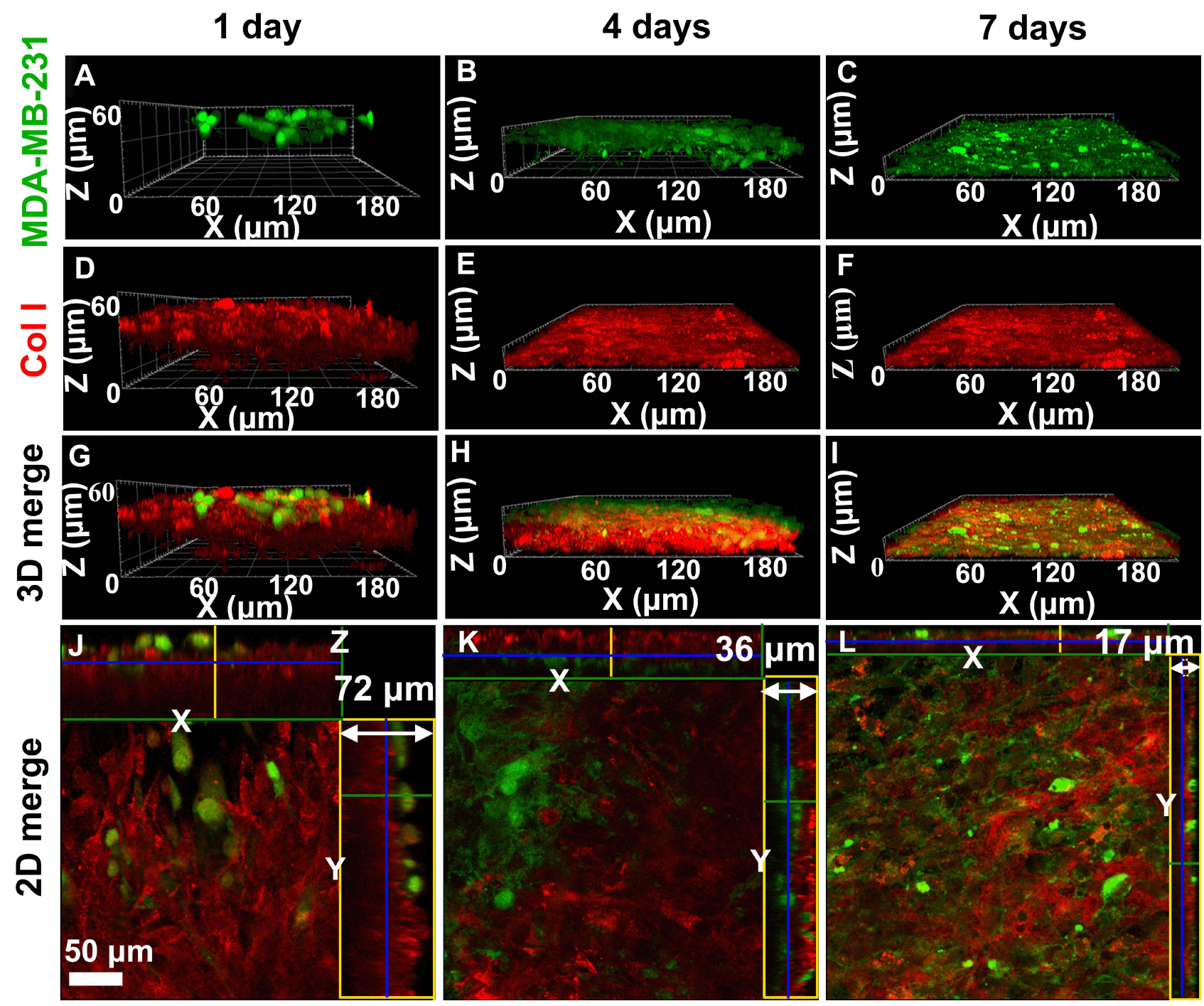

Figure 2 3D dynamic observation (A-I) and the 2D section morphologies (J-L) of cancer cells (green) and the cell-secreted Col I matrix (red) during bone metastasis of breast cancer in the 3D engineered bone matrix model via LCSM. (A, D, G, J) Co-cultured for I day; (B, E, H, K) co-cultured for 4 days; (C, F, I, L) co-cultured for 7 days. Bar $=50 \mu \mathrm{m}$.

thickness of the matrix decreased from $72 \mu \mathrm{m}$ to $17 \mu \mathrm{m}$ with the culture developing. The control sample of the mineralized bone matrix without breast cancer cells on the 7th day was added in Figure S2. The thickness of the matrix co-cultured with/without breast cancer cells for the 7th day was also showed in Figure S2C and there was only a slight increase in the thickness of the matrix co-cultured without breast cancer cells (no statistical difference, $\mathrm{P}>0.05$ ). We hypothesized breast cancer cells would destruct the bone matrix and then the broken matrices were lost as the medium was changed.

Figure 3 shows the morphologies and migration of breast cancer cells in the bone matrix. After the MDA-MB-231 cells co-cultured with the bone matrix for 1 day (Figure $3 \mathrm{~A}$ and E), the MDA-MB-231 cells grew into the matrix, distributing in the engineered bone matrix. As the culture time increased, breast cancer cells stretched along the alignment of collagen fibrils of bone matrix and invaded longitudinally along $\mathrm{Z}$ axis (Figure $3 \mathrm{~B}$ and $\mathrm{F}$ ). Fiber orientation distribution of Col I matrix and the breast cancer cell has been shown in Figure 3I-L. As the breast cancer cells stretched in orientation, the collagen matrix became sufficiently loose and reduced thickness after 5-day culture as shown in Figure 3C and G. When the breast cancer cells are completely infiltrated into the matrix, the pseudopods of the breast cancer cells are distributed throughout the matrix and the thickness of the stroma was reduced to a minimum one (7-day co-culture, Figure 3D and $\mathrm{H}$ ). As shown in Figures 2 and 3, bone matrix secreted by osteoblasts is broken and the longitudinal invasion of breast cancer cells was observed. 


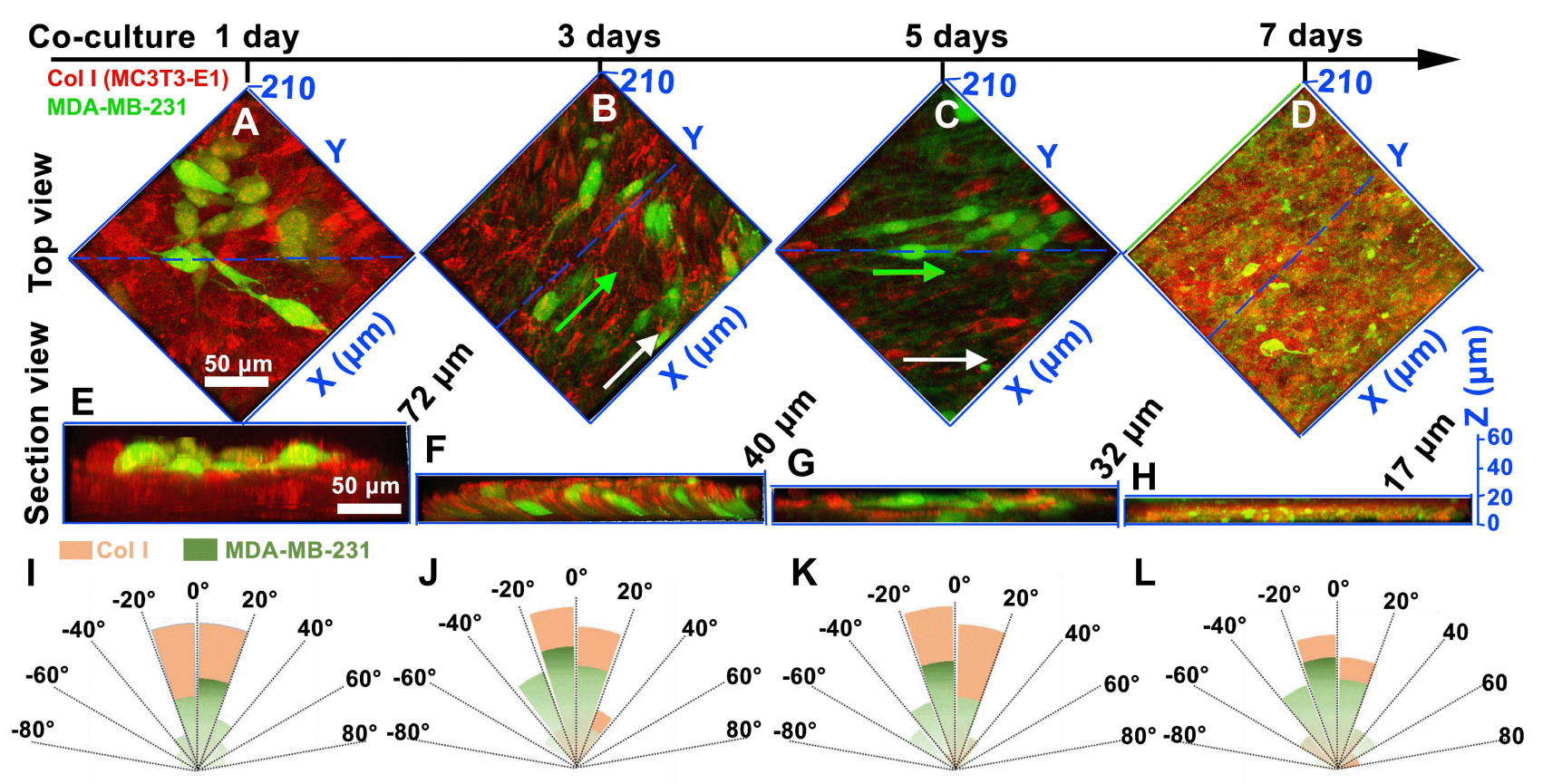

Figure 3 The top views (A-D) and the 2D section morphologies (E-H) of breast cancer cells (in green) and the cell-secreted Col I matrix (in red) via LCSM. (A and E) Cocultured for I day; (B and F) co-cultured for 3 days; ( $\mathbf{C}$ and $\mathbf{G}$ ) co-cultured for 5 days; ( $\mathbf{D}$ and $\mathbf{H})$ co-cultured for 7 days. The white arrows indicate the orientation of the Col I matrix. The green arrow is the orientation of the breast cancer cell. The dashed blue line is the interception plane of the section view images. (I-L) Fiber orientation distribution of Col I matrix and the breast cancer cell. The results showed that breast cancer cells extended along the orientation of collagen fibers in the early stage of cell co-culture $(\mathbf{J}$ and $\mathbf{K})$. As breast cancer cells invade the mineralized matrix, the orientation of both collagen fibers and breast cancer cells decreases.
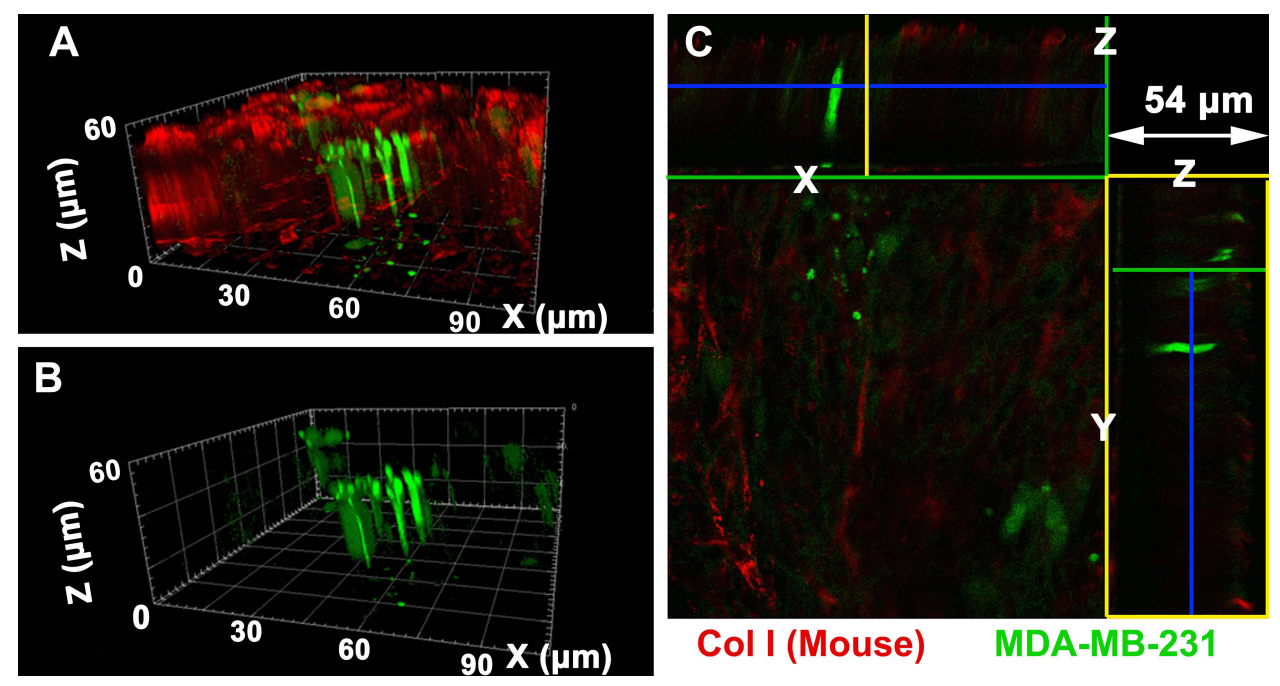

Figure 4 Morphologies of breast cancer cells co-cultured for 3 days during bone metastasis observed via LCSM and it shows that the cancer cells in long spindle shape infiltrated into the Col I matrix. (A) 3D morphologies of cancer cells and collagen fiber in the bone matrix; (B) 3D morphologies of cancer cells; (C) 2D section morphologies of cancer cells co-cultured with the cell-secreted Col I matrix.

To explore the longitudinal invasion of breast cancer cells in the matrix, the morphologies of MDA-MB-231 cells co-cultured for 3 days in the engineered bone matrix could be clearly observed in Figure 4. The morphologies of MDA-MB-231 cells can also be clearly observed in the $\mathrm{X}-\mathrm{Z}$ cross-section (green box in Figure $4 \mathrm{C}$ ) and $\mathrm{Y}-\mathrm{Z}$ crosssection (yellow box in Figure 4C) that the cancer cells infiltrated into the matrices in long spindle shapes along $\mathrm{Z}$ axis, which keep the same direction as shown in Figure 4A. An arbitrary height was selected from the 3D reconstructed model to analyze the internal details of the matrix (Figure 4C). We could find that the cancer cells were appearing elongated and infiltrating the matrix along a downward way along $\mathrm{Z}$ axis. 

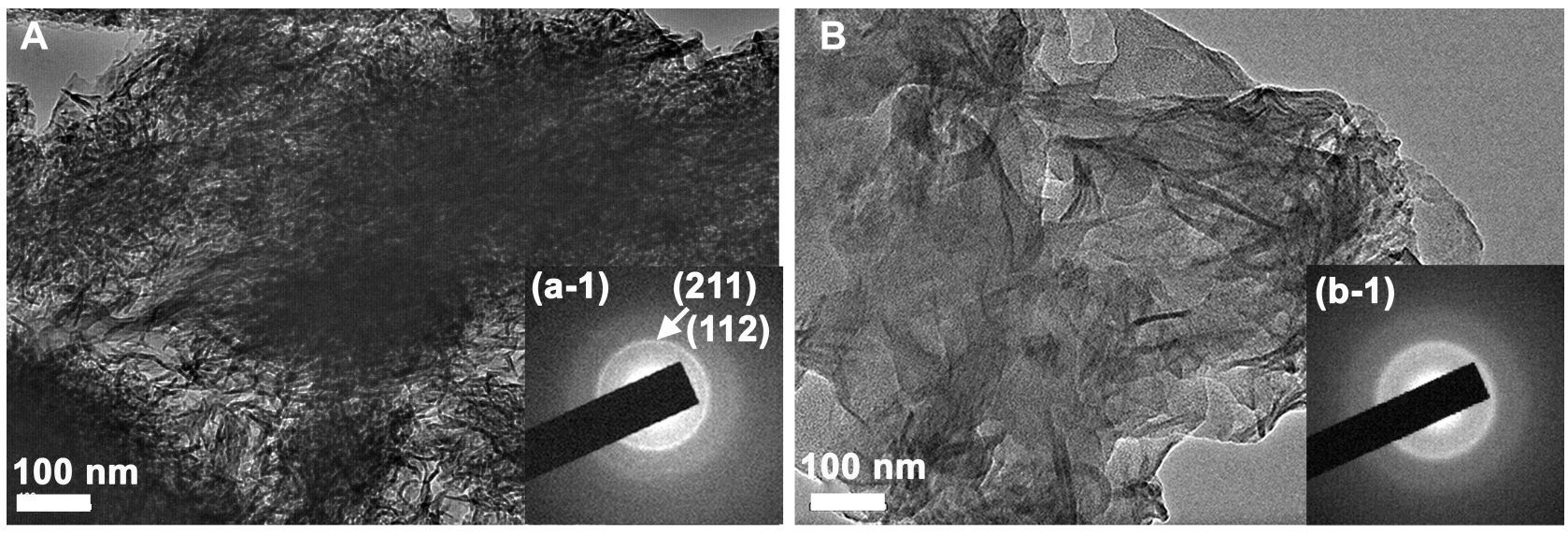

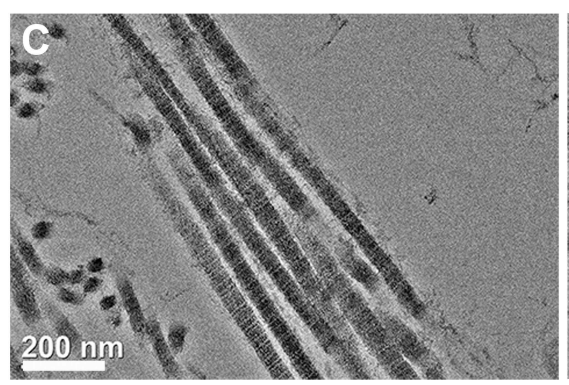

$\mathbf{F}$

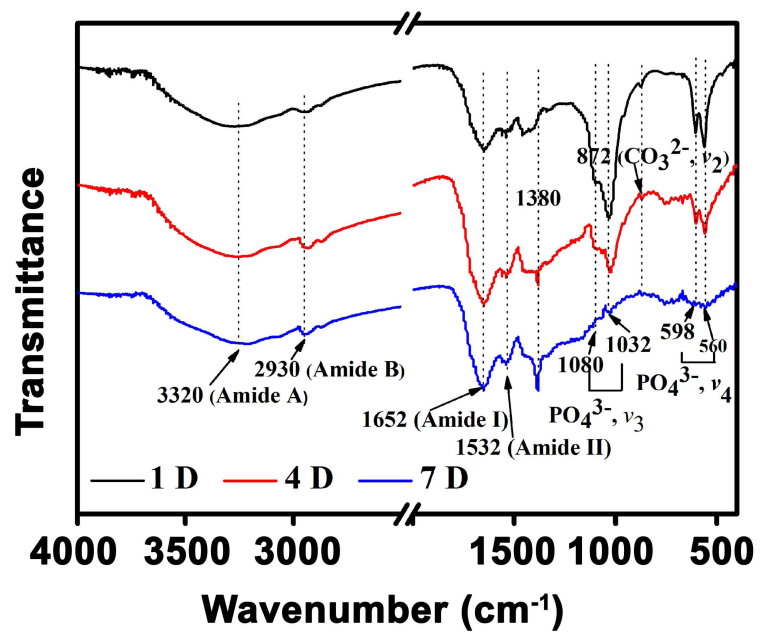

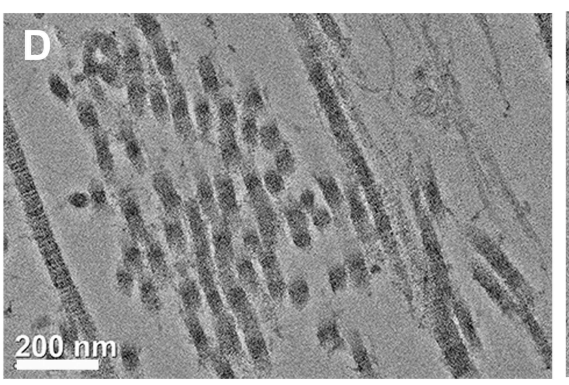

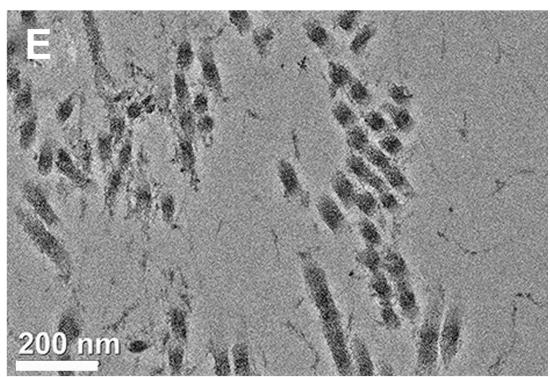

G

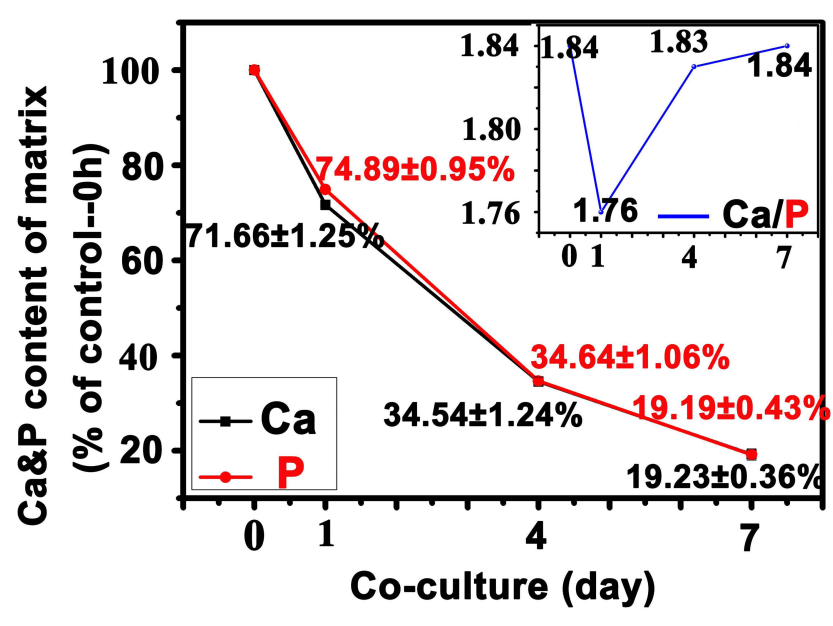

Figure 5 Characterization of the engineered bone matrix with/without breast cancer cells co-culturing. TEM (A and B) and SAED (a-I, b-I) images of the mineralized collagen fibril bundles before cancer cells seeded $(\mathbf{A})$ and after 7-day co-culturing (B); TEM images of uranyl acetate-stained mineralized collagen fibril bundles for I-day (C), 4-day (D) and 7-day (E) co-culturing; (F) FTIR spectra and (G) the contents of Ca and P of the engineered bone matrix with breast cancer cells co-culturing.

\section{Analysis of Ultrastructure of Bone Metastasis}

Collagen fibrils in the engineered bone matrix before and after cancer cell co-culture were observed by TEM (Figure 5A and B). Collagen fibrils in the matrix are clustered, arranged in a dense and orderly manner, and assembled into bundles before cancer cells were seeded (Figure 5C). There are characteristic diffraction rings of
(211) and (112) crystal planes of HAP in the polycrystalline electron diffraction pattern. However, the diffraction rings are relatively unclear due to the weak crystallization of HAP. After 7-day cell culture, collagen fibrils decreased in a large amount and were arranged in an extremely loose and disordered manner, and the fibril also decreased significantly in length (Figure 5E). At the same time, the characteristic diffraction rings of HAP became blurred, 
which means the crystal structure of HAP has been damaged.

The ultrastructure of the matrix is shown in Figure 5C-E. On the initial day, the collagen fibrils of the matrix were assembled into bundles in long strips as shown in Figure 5C, and the D-period banding of collagen fibrils was distinguished. No obvious minerals can be detected in the view, which means HAP might be formed via intrafibrillar mineralization. The results once again proved that the matrix was similar to the native bone structure. With the development of incubation, collagen fibrils in the model were seriously encroached by cancer cells. The fibrils were truncated, and their length was decreased and even broken into pieces in TEM (Figure 5D). After 7 days, the collagen fibrils were broken into short clusters and no apparent assembled structure was observed, indicating that the ordered structure of the self-assembled collagen fibrils had been seriously destroyed (Figure 5E). Before cancer cells be seeded, collagen fibrils in the model packed into aligned morphology. After cancer cells co-cultured with osteoblast in the bone matrix, the assembled structure of collagen fibrils was damaged. So, the ordered structure of the bone matrix was destroyed. Obviously, the typical assembled structure of collagen can no longer be distinguished at this time, indicating that breast cancer cells may erode and decompose the collagen matrix in the process of bone metastasis, thus leading to the osteolytic destruction of bone tissue.

FTIR spectra and ICP-MS were used to analyze the composition of bone matrix during cell culture (Figure 5F and G). The characteristic absorption peaks of organic collagen did not change much. The characteristic peak position of $\mathrm{PO}_{4}{ }^{3-}$ in HAP was still around $1032 \mathrm{~cm}^{-1}$, and the characteristic peak position of $\mathrm{Ca}-\mathrm{O}$ was near the $513 \mathrm{~cm}^{-1}$. With cell culture processing, the peak strength of the two characteristic absorption peaks gradually decreased, and the absorption peaks tended to be flat after 7 days, showing that the content of HAP in the matrix decreased. Moreover, the concentrations of $\mathrm{Ca}$ and $\mathrm{P}$ ions gradually decreased with cell culture, suggesting that inorganic substances such as HAP in the bone model were engulfed or degraded by cancer cells, proving that the occurrence of bone metastasis caused severe bone erosion and osteolysis.

\section{Discussion}

\section{D Engineered Bone Matrix Formation via Cell Biomineralization on Template}

Bone is the most prevalent tissue for breast cancer metastasis and about $73 \%$ of breast cancer patients suffer from osteolytic bone metastases. ${ }^{15}$ However, the role of bone ECM in cancer progression is still unknown. The bone environment is comprised of complex and dynamic interactions between resident matrix and cells, all of which are related to the pathogenesis of bone cancers. Different 3D models have been made to mimic bone matrix and study bone microenvironment and metastasis from breast cancer. ${ }^{19,35}$ Several approaches have been developed to construct collagen fibrous architectures in vitro to mimic the physicochemical environment of the bone tumor niche. ${ }^{36,37}$ These methods can prepare oriented collagen fiber scaffolds with certain characteristics of bone collagen. However, preparing the oriented mineralized collagen structure is challenging, where HAP acts as the component to support tissue and maintain normal activities. ${ }^{29}$ In the study, we used an osteoid-like collagen template with typically oriented structure to guide cells' behavior and further constructed a 3D engineered bone matrix via cell mineralization. According to Figure 1, the 3D mineralized ECM secreted by cells contains orientated collagen fibers and an inorganic product of HAP. The matrix was characterized at multiple levels to verify the formation of a 3D engineered bone matrix with this approach. First, mineralized matrices were stained using VK staining to detect phosphate compounds that indicate mineral formation (Figure 1D). The result revealed positive staining in cell-produced ECM when osteoblasts were cultured on the template. In contrast, the collagen template showed no calcium nodules. Then, the chemical composition of the matrix was analyzed by FTIR spectra (Figure 1I) and the mineral phase was analyzed by XRD (Figure 1H). XRD and FTIR spectra showed HAP characteristic peaks in the matrix. It can then be seen from the SEM images that the collagen fibers in the ecm produced by the cells are aligned in the direction of the collagen fibers in the template. Further, as shown in Figure 5C, the produced dense assembled collagen fibrils packed into paralleling pattern, which allowed for easier diffusion of the mineral particles, usually termed intrafibrillar mineralization. ${ }^{38}$ In summary, these findings indicate that templateguided bone matrix generates compositionally and structurally relevant bone microenvironment, which could be used to examine tumor cell behavior as a function of bone matrix. 


\section{Bone Osteolytic Process in Breast Cancer Bone Metastasis}

Some studies have shown that anisotropy enhances the invasion of cancer cells into the surrounding ECM and supports their ultimate metastasis. ${ }^{39,40}$ At the same time, the aligned collagen bundles, which mimic the structure of tumor-related collagen fibers, have been shown to trigger cancer cell contact guidance and enhance directional migration. $^{41,42}$ Bio-interface anisotropy regulated the cell's morphology and mediated the migration pattern. Morphologically, the cells on anisotropic nanofibers showed an elongated spindle shape. ${ }^{43} 3 \mathrm{D}$ aligned collagen matrices enhanced the directional migration of cancer stem cells. ${ }^{44}$ As shown in Figures 2-4, engineered 3D bone-like matrix with aligned mineralized collagen fibrils is easy to recruit MDA-MB-231-GFP breast cancer cells and the cancer cells are stretched in an elongated shape aligned to the mineralized collagen fibrils and infiltrated into the matrices, initiating the degradation of bone matrix and leading to osteolysis.

Bone is the most frequent site of breast cancer metastasis, yet why these cancer cells preferentially metastasize to bone remains largely unclear. Oriented collagen scaffolds have been used as a model to culture breast cancer cells, showing that culturing in a $3 \mathrm{D}$ model induces lasting changes in cell behavior. Meanwhile, intrafibrillar HAP decreased the adhesion forces of breast cancer cell and accordingly reduced the collagen fiber arrangement relative to cell culture on the controlled collagen. ${ }^{4}$ These developed substrates cannot recapitulate the complexity of bone ECM. Herein, cell-deposited bone ECMs guided by the template can provide a platform for studying cellcell and cell-material interactions and more closely recapitulate the pathophysiological features of native metastatic tissues. ${ }^{35,45}$ In addition, the bone matrix can test how breast cancer cells destroy bone tissue, and there are few related reports.

According to the above results, after co-culture of cancer cells with osteoblast in the bone matrix, the ordered structure of collagen fibrils was damaged by cancer cells. What is more, the typical assembled structure of mineralized collagen fibrils was eroded and the bone matrix decomposed in the process of co-culture. The loss of mineral also is a typical symptom of osteolytic bone metastasis. For the first time, this phenomenon is simulated in an in vitro $3 \mathrm{D}$ model. In contrast to other mineral-containing collagen matrices, the aligned fibrils with intrafibrillar mineral, achieved via cell mineralization, more accurately recapitulates the compositional and structural feature of bone. So, the biomimetic platform provides the possibility to study the mechanism for cancer-bone metastasis.

ECM degradation is a critical step in cancer cell invasion and is important for local migration and escape of tumors in the early stages of metastasis. ${ }^{12}$ MDA-MB-231 breast cancer cells can cause the loss of the collagen network. Cancer cells produce proteinases, including matrix metalloproteinases (MMPs) and plasminogen activator system (PAS) which promote cancer cell invasion of extracellular matrices. ${ }^{46}$ In vivo, cancer cells secrete proteolytic enzymes to remodel and degrade the ECM and are polarized to move toward blood vessels. ${ }^{47}$ Some studies had discovered that cancer-bearing bone showed the disorganized, less-aligned collagen/apatite microstructure. ${ }^{48}$ The apatite crystals of ex vivo tumorinvaded bone showed a significantly disrupted arrangement. ${ }^{49}$ We hypothesized that a tumor microenvironment was achieved in this simulated bone metastasis, where typical disruption of assembled collagen matrix is attributed to the osteolytic bone metastasis.

\section{Conclusion}

In this study, an engineered 3D bone-like matrix was successfully prepared via cell mineralization guided by an osteoid-like template. The bone-like matrices are composed of mineralized collagen fibril bundles. Then, breast cancer cells and the matrix were co-cultured to simulate the process of breast cancer bone metastasis. The cancer cells spread in an elongated way along the alignment of collagen fibrils matrix and infiltrated to invade the matrix vertical to the alignment of collagen fibrils. With the development of co-culture, the bone matrix was eroding, altogether with Col I and HAP. Also, mineralized collagen fibrils bundles were broken into pieces and the oriented structure was seriously eroded. The finding revealed the process of bone metastasis relative to breast cancer, where mineralized collagen fiber disassembled at nanoscale. The interaction between osteoblasts, breast cancer cells and bone matrix microenvironment were simulated in vitro through this model. We believe that our study is beneficial to study the pathogenesis of bonerelative disease and to further explore the new therapy to cancer bone metastasis.

\section{Acknowledgments}

This work was financially supported by the National Natural Science Foundation of China (grant numbers 31971284). 


\section{Disclosure}

The authors report no conflicts of interest for this work.

\section{References}

1. Li C, Hu J, Li W, et al. Combined bortezomib-based chemotherapy and $\mathrm{p} 53$ gene therapy using hollow mesoporous silica nanospheres for p53 mutant non-small cell lung cancer treatment. Biomater Sci. 2016;5:77-88. doi:10.1039/C6BM00449K

2. Hess KR, Varadhachary GR, Taylor SH, et al. Metastatic patterns in adenocarcinoma. Cancer. 2006;106:1624-1633. doi:10.1002/cncr.21778

3. Croucher PI, McDonald M, Martin TJ. Bone metastasis: the importance of the neighbourhood. Nat Rev Cancer. 2016;16:373-386.

4. Choi S, Friedrichs J, Song YH, et al. Bone-mimetic collagen mineralization regulates breast cancer cell adhesion and migration. Biomaterials. 2019;18:95-106. doi:10.1016/j.biomaterials.2018.05.002

5. Akhshi TK, Wernike D, Piekny A. Microtubules and actin crosstalk in cell migration and division. Cytoskeleton. 2014;71:1-23. doi: $10.1002 / \mathrm{cm} .21150$

6. Mundy GR. Metastasis to bone: causes, consequences and therapeutic opportunities. Nat Rev Cancer. 2002;2:584-593. doi:10.1038/nrc867

7. Liu J, Zeng Y, Shi S, et al. Design of polyaspartic acid peptide-poly (ethylene glycol)-poly (epsilon-caprolactone) nanoparticles as a carrier of hydrophobic drugs targeting cancer metastasized to bone. Int J Nanomedicine. 2017;12:3561-3575. doi:10.2147/IJN. S133787

8. Pandya M, Diekwisch TGH. Enamel biomimetics-fiction or future of dentistry. Int J Oral Sci. 2019;11:8. doi:10.1038/s41368-018-0038-6

9. Cox RF, Morgan MP. Microcalcifications in breast cancer: lessons from physiological mineralization. Bone. 2013;53:437-450. doi:10.1016/j.bone.2013.01.013

10. Mastr AM, Gay CV, Welch DR. The skeleton as a unique environment for breast cancer cells. Clin Exp Metastas. 2003;20:275-284. doi:10.1023/A:1022995403081

11. Bussard KM, Okita N, Sharkey N, et al. Localization of osteoblast inflammatory cytokines MCP-1 and VEGF to the matrix of the trabecula of the femur, a target area for metastatic breast cancer cell colonization. Clin Exp Metastasis. 2010;27:331-340. doi:10.1007/ s10585-010-9330-3

12. Zhang Y, Ma B, Fan Q. Mechanisms of breast cancer bone metastasis. Cancer Lett. 2010;292:1-7. doi:10.1016/j. canlet.2009.11.003

13. Mastro AM, Gay CV, Welch DR, et al. Breast cancer cells induce osteoblast apoptosis: a possible contributor to bone degradation. J Cell Biochem. 2004;91:265-276. doi:10.1002/jcb.10746

14. Wang M, Xia F, Wei Y, et al. Molecular mechanisms and clinical management of cancer bone metastasis. Bone Research. 2020;8:30. doi:10.1038/s41413-020-00105-1

15. Guise TA, Mohammad KS, Clines G, et al. Basic mechanisms responsible for osteolytic and osteoblastic bone metastases. Clin Cancer Res. 2006;12:6213s-6216s. doi:10.1158/1078-0432.CCR-06-1007

16. Zhu W, Wang M, Fu Y, et al. Engineering a biomimetic three-dimensional nanostructured bone model for breast cancer bone metastasis study. Acta Biomater. 2015;14:164-174. doi:10.1016/j.actbio.2014.12.008

17. Mercatali L, Spadazzi C. The effect of everolimus in an In vitro model of triple negative breast cancer and osteoclasts. Int $\mathrm{J} \mathrm{Mol} \mathrm{Sci.}$ 2016;17:1827. doi:10.3390/ijms17111827

18. Mercatali L, La Manna F, Miserocchi G, et al. Tumor-stroma crosstalk in bone tissue: the osteoclastogenic potential of a breast cancer cell line in a co-culture system and the role of EGFR Inhibition. Int J Mol Sci. 2017;1:18.

19. Qiao H, Tang T. Engineering 3D approaches to model the dynamic microenvironments of cancer bone metastasis. Bone Res. 2018;6:3. doi:10.1038/s41413-018-0008-9
20. Haider MT, Zarrer J, Taipaleenmäki H. Breast cancer bone metastases are attenuated in a Tgifl-deficient bone microenvironment. Breast Cancer Res. 2020;2:324-341.

21. McGonigle P, Ruggeri B. Animal models of human disease: challenges in enabling translation. Biochem Pharmacol. 2014;87:162-171. doi:10.1016/j.bcp.2013.08.006

22. Su W, Ma L, Ran Y, et al. Alginate-assisted mineralization of collagen by collagen reconstitution and calcium phosphate formation. ACS Biomater Sci Eng. 2020;6:3275-3286. doi:10.1021/ acsbiomaterials.9b01841

23. Wingender B, Bradley P, Saxena N, et al. Biomimetic organization of collagen matrices to template bone-like microstructures. Matrix Biol. 2016;52-54:384-396. doi:10.1016/j.matbio.2016.02.004

24. Aveic S, Davtalab R, Vogt M, et al. Calcium phosphate scaffolds with defined interconnecting channel structure provide a mimetic 3D niche for bone marrow metastasized tumor cell growth. Acta Biomater. 2019;88:527-539. doi:10.1016/j.actbio.2019.02.030

25. Redmond J, McCarthy H, Buchanan P, et al. Advances in biofabrication techniques for collagen-based 3D in vitro culture models for breast cancer research. Mat Sci Eng C-Mater. 2021;122:111944. doi:10.1016/j.msec.2021.111944

26. Mapanao AK, Voliani V. Three-dimensional tumor models: promoting breakthroughs in nanotheranostics translational research. Appl Mater Today. 2020;19:100552. doi:10.1016/j.apmt.2019.100552

27. Siolas D, Hannon GJ. Patient-derived tumor xenografts: transforming clinical samples into mouse models. Cancer Res. 2013;73:5315-5319. doi:10.1158/0008-5472.CAN-13-1069

28. Walsh NC, Kenney LL, Jangalwe S, et al. Humanized mouse models of clinical disease. Annu Rev Pathol. 2017;12:187-215. doi:10.1146/ annurev-pathol-052016-100332

29. Ma C, Wang H, Chi Y, et al. Preparation of oriented collagen fiber scaffolds and its application in bone tissue engineering. Appl Mater Today. 2021;22:100902-100915. doi:10.1016/j. apmt.2020.100902

30. Luo X, Zhang S, Luo B, et al. Engineering collagen fiber templates with oriented nanoarchitecture and concerns on osteoblast behaviors. Int J Biol Macromol. 2021;185:77-86. doi:10.1016/j.ijbiomac.20 21.06.072

31. Matsugaki A, Ozasa R, Isobe Y, et al. Oriented collagen scaffolds for anisotropic bone tissue construction in vitro. Mater Sci Forum. 2014;783-786:1303-1306. doi:10.4028/www.scientific.net/MSF.783786.1303

32. Casale C, Imparato G, Mazio C, et al. Geometrical confinement controls cell, ECM and vascular network alignment during the morphogenesis of 3D bioengineered human connective tissues. Acta Biomater. 2021;131:341-354. doi:10.1016/j.actbio.2021.06.022

33. Rusu VM, Ng CH, Wilke M, et al. Size-controlled hydroxyapatite nanoparticles as self-organized organic-inorganic composite materials. Biomaterials. 2005;26:5414-5426. doi:10.1016/j. biomaterials.2005.01.051

34. Wan C, Chen B. Synthesis and characterization of biomimetic hydroxyapatite/sepiolite nanocomposites. Nanoscale. 2011;3:693-700. doi:10.1039/C0NR00650E

35. Salamanna F, Contartese D, Maglio M, et al. A systematic review on in vitro $3 \mathrm{D}$ bone metastases models: a new horizon to recapitulate the native clinical scenario? Oncotarget. 2016;7:44803-44820. doi:10.18632/oncotarget.8394

36. Rieu CM, Parisi C, Mosser G, et al. Topotactic fibrillogenesis of freeze-cast microridged collagen scaffolds for $3 \mathrm{D}$ cell culture. ACS Appl Mater Interfaces. 2019;11:14672-14683. doi:10.1021/ acsami.9b03219

37. Luo X, Guo Z, He P, et al. Study on structure, mechanical property and cell cytocompatibility of electrospun collagen nanofibers crosslinked by common agents. Int J Biol Macromol. 2018;113:476-486. doi:10.1016/j.ijbiomac.2018.01.179 
38. Niu LN, Jee SE, Jiao K, et al. Collagen intrafibrillar mineralization as a result of the balance between osmotic equilibrium and electroneutrality. Nat Mater. 2017;16:370-378. doi:10.1038/ nmat4789

39. Conklin MW, Keely PJ. Why the stroma matters in breast cancer: insights into breast cancer patient outcomes through the examination of stromal biomarkers. Cell Adh Migr. 2012;6:249-260. doi:10.4161/ cam. 20567

40. Provenzano PP, Inman DR, Eliceiri KW, et al. Collagen density promotes mammary tumor initiation and progression. BMC Med. 2008;6:11. doi:10.1186/1741-7015-6-11

41. Gong X, Kulwatno J, Mills KL. Rapid fabrication of collagen bundles mimicking tumor-associated collagen architectures. Acta Biomater. 2020;108:128-141. doi:10.1016/j.actbio.2020.03.019

42. Ray A, Provenzano PP. Aligned forces: origins and mechanisms of cancer dissemination guided by extracellular matrix architecture. Curr Opin Cell Biol. 2021;72:63-71. doi:10.1016/j.ceb.2021.05.004

43. Dai J, Wang Y, Gong J, et al. Biointerface anisotropy modulates migration of breast cancer cell. Colloid Surf B Biointerfaces. 2020;190:110973. doi:10.1016/j.colsurfb.2020.110973
44. Ray A, Slama ZM, Morford RK, et al. Enhanced directional migration of cancer stem cells in 3D aligned collagen matrices. Biophys $J$. 2017;112:1023-1036. doi:10.1016/j.bpj.2017.01.007

45. Sitarski AM, Fairfield H, Falank C, et al. 3D tissue engineered in vitro models of cancer in bone. ACS Biomater Sci Eng. 2018;4:324-336. doi:10.1021/acsbiomaterials.7b00097

46. Morgan H, Hill PA. Human breast cancer cell-mediated bone collagen degradation requires plasminogen activation and matrix metalloproteinase activity. Cancer Cell Int. 2005;5:1. doi:10.1186/ 1475-2867-5-1

47. Condeelis J, Segall JE. Intravital imaging of cell movement in tumours. Nat Rev Cancer. 2003;3:921-930. doi:10.1038/nrc1231

48. Sekita A, Matsugaki A, Nakano T. Disruption of collagen/apatite alignment impairs bone mechanical function in osteoblastic metastasis induced by prostate cancer. Bone. 2017;97:83-93.

49. Matsugaki A, Harada T, Kimura Y, et al. Dynamic collision behavior Between osteoblasts and tumor cells regulates the disordered arrangement of collagen fiber/apatite crystals in metastasized bone. Int J Mol Sci. 2018;19:3474. doi:10.3390/ijms19113474
International Journal of Nanomedicine

\section{Publish your work in this journal}

The International Journal of Nanomedicine is an international, peerreviewed journal focusing on the application of nanotechnology in diagnostics, therapeutics, and drug delivery systems throughout the biomedical field. This journal is indexed on PubMed Central, MedLine, CAS, SciSearch ${ }^{\circledR}$, Current Contents ${ }^{\circledR} /$ Clinical Medicine,
Journal Citation Reports/Science Edition, EMBase, Scopus and the Elsevier Bibliographic databases. The manuscript management system is completely online and includes a very quick and fair peer-review system, which is all easy to use. Visit http://www.dovepress.com/ testimonials.php to read real quotes from published authors. 